(43.6\%) were on diuretics mainly for hypertension $(64.7 \%)$, heart failure $(9.4 \%)$, and renal failure $(5.9 \%)$. Follow-up data was available from 209 patients $(92.9 \%)$, with a median 9 months of follow-up (4-14). ULT used was allopurinol in 172 patients $(82.6 \%)$, febuxostat in $34(16.5 \%)$, and benzbromarone in only 2 cases $(0.9 \%)$. Regarding the baseline characteristics, patients on diuretics were older, had higher rates of females, hypertension, diabetes, and cardiovascular disease, and showed higher SUA and lower glomerular filtration rate. The table shows the outcomes comparison according to diuretic treatment, globally and stratified by type of ULT (excluding the two cases of benzbromarone). Except for a lower achievement of $\mathrm{SUA}<5$ in the allopurinol subgroup, no significant differences were found either globally or by type of ULT.

Conclusions: Despite its high rate, diuretics currently seem to not have a significant impact on the achievement of the different objectives of treatment of patients with gout.

References:

[1] Rheumatology; 55:1172.

[2] Br J Clin Pharmacol; 81:277.

Disclosure of Interest: None declared

DOI: 10.1136/annrheumdis-2017-eular.3905

\section{THU0438 LACK OF PREDICTIVE VALUE OF THE NIAID/FAAN CRITERIA TO IDENTIFY SUBJECTS WITH EVIDENCE OF IMMUNE ACTIVATION AFTER RECEIVING PEGLOTICASE FOR CHRONIC REFRACTORY GOUT}

L. Calabrese ${ }^{1}$, A. Kavanaugh ${ }^{2}$, A. Yeo ${ }^{3}$, P. Lipsky ${ }^{4} .{ }^{1}$ Rheumatic and Immunologic Diseases, Cleveland Clinic, Cleveland, $\mathrm{OH}^{2}{ }^{2}$ Division of Rheumatology, Allergy, \& Immunology, UCSD, la Jolla, CA; ${ }^{3}$ Horizon Pharma, Lake Forest, IL; ${ }^{4}$ AMPEL BioSolutions, LLC, Charlottesville, VA, United States

Background: The NIAID/FAAN criteria have been widely applied to identify anaphylaxis despite having only modest specificity. 1,2

Objectives: To evaluate the predictive value of the NIAID/FAAN criteria to identify evidence of immune activation in subjects with chronic refractory gout treated with pegloticase in randomized clinical trials (RCTS) who experienced infusion reactions (IRs), by assessing the capacity of biochemical testing for immunologic activation to confirm the likelihood of anaphylaxis.

Methods: During the RCTs supporting the approval of pegloticase for treatment of chronic refractory gout, subjects remained on therapy despite becoming nonresponders, which reflected the development of anti-drug antibodies (ADA); $\sim 60 \%$ of subjects continued to receive pegloticase despite elevated ADA titers and many had IRs. In subjects receiving pegloticase every 2 weeks (q2w), 22/85 (25.9\%) had a total of 43 IRs and $34 / 84(40.5 \%)$ receiving pegloticase every 4 weeks (q4w) had a total of 70 IRs. $21 / 22(95.5 \%)$ and $29 / 34$ (85.3\%) subjects with IRs in the $\mathrm{q} 2 \mathrm{w}$ and $\mathrm{q} 4 \mathrm{w}$ groups, respectively, were nonresponders. The 113 total IRs were categorized post hoc as to whether they met NIAID/FAAN criteria for anaphylaxis. Six IRs in 6 subjects met criteria, 53 IRs in 33 subjects had only one feature and were designated as "hypersensitivity", and 54 IRs in 29 subjects had no features and were designated "other". The clinical courses of these IRs and whether they were associated with elevated tryptase levels as a measure of mast cell degranulation or complement consumption as a measure of immune complex activity were assessed.

Results: Of a total of 852 infusions in subjects receiving q2w pegloticase, 43 $(5.0 \%)$ were associated with IRs as were 70 of 830 infusions (9.5\%) in subjects receiving $\mathrm{q} 4 \mathrm{w}$ pegloticase. Of the $70 \mathrm{IRs}$ associated with $\mathrm{q} 4 \mathrm{w}$ administration, 3 $(4.3 \%)$ met the criteria for anaphylaxis, $31(44.3 \%)$ for hypersensitivity and 36 $(51.4 \%)$ were other. The respective values for subjects receiving pegloticase q2w were $3 / 43(7.0 \%), 22 / 43(51.1 \%)$, and $18 / 43(41.9 \%)$. A total of 14 IRs $(12.4 \%)$ were associated with elevated tryptase and $31(27.4 \%)$ with decreased complement $\mathrm{CH} 50$ levels. Immunologic abnormalities were not significantly different in the 3 groups, with $16.7 \%, 17.0 \%$ and $7.4 \%$ of anaphylaxis, hypersensitivity and other IRs, respectively, having increased tryptase; and $0 \%, 30.2 \% \%$, and $27.8 \%$, respectively, having decreased complement. No subjects were classified as experiencing anaphylaxis by investigators, none required hospitalization, and $60.7 \%$, continued to receive pegloticase.

Conclusions: Post-hoc designation of pegloticase-treated subjects as meeting NIAID/FAAN criteria for anaphylaxis was not associated with a higher frequency of biochemical evidence of immune activation or a more severe clinical course. In pegloticase-treated subjects, the NIAID/FAAN anaphylaxis criteria did not identify subjects with IRs associated with elevated tryptase and decreased complement $\mathrm{CH} 50$ levels.

References:

[1] Sampson HA, et al. J Allergy Clin Immunol. 2006;117:391-397.

[2] Campbell RL, et al. J Allergy Clin Immunol. 2012;129:748-752.

Disclosure of Interest: L. Calabrese Consultant for: BMS, Genentech, Horizon, Crescendo, Pfizer, GSK, Regeneron, UCB, Abbvie, Janssen, A. Kavanaugh Consultant for: Abbvie, Amgen, Janssen, BMS, UCB, Pfizer, A. Yeo Consultant for: Horizon Pharma, P. Lipsky Consultant for: AstraZeneca, Celgene, EMD Serono, GSK, Horizon Pharma, Janssen, Medimmune, Pfizer, Roche, Sanofi, UCB

DOI: 10.1136/annrheumdis-2017-eular.3906

\section{THU0439 MORE OBESITY AND HIGHER SERUM URIC ACID IN EARLY-ONSET GOUT PATIENTS IN SOUTH CHINA}

Q.-H. Li ${ }^{1}$, L.-X. Chen ${ }^{2}$, Y.-H. Li ${ }^{1}$, D.-H. Zheng ${ }^{1}$, Y.-Q. Mo ${ }^{1}$, L. Dai ${ }^{1}$, J.-J. Liang ${ }^{1}$, X.-N. Wei ${ }^{1} .{ }^{1}$ Department of Rheumatology; ${ }^{2}$ Department of Internal Medecine, Sun Yat-Sen Memorial Hospital, Sun Yat-Sen University, Guangzhou, China

Background: Gout incidence increases in a linear fashion with age until 70 years. It was reported that there was a trend of earlier gout onset. Few studies about clinical features of early-onset gout in China are reported.

Objectives: To investigate the characteristics of early-onset gout in south China. Methods: Patients with primary gout were recruited between June 2012 and December 2016. Face to face interview was carried out to collect demographic information, clinical characteristics of gout and comorbid diseases. The patients with gout onset before age 30 were defined as early-onset group. The other gout patients were enrolled as control group.

Results: (1) Five hundred and one gout patients were recruited, including 99 $(19.8 \%)$ in the early-onset group and $402(80.2 \%)$ in the control group. The onset age of the early-onset group was $24.2 \pm 5.8$ (range 14-30) and the control group was $51.7 \pm 13.9$ (range 31-84). There was no significant difference of gout duration between early-onset group and control group $(P<0.05$, Table 1). (2) Compared with the control group, the early-onset group presented lower frequency of flare in last year, higher serum uric acid and higher proportion of $s \cup A>10 \mathrm{mg} / \mathrm{dl}(P<0.05$, Table 1). Body mass index (BMI) and prevalence of obesity were higher in the early-onset group $(P<0.05$, Table 1$)$. The early-onset group exhibited lower prevalence of hypertension, diabetic mellitus, urolithiasis and chronic kidney disease (CKD) $(P<0.05$, Table 1). (3) There were 20 patients (20.2\%) with tophi in the early-onset group. Compared with the 79 early-onset gout patients without tophi, the patients with tophi presented higher age $(39.6 \pm 8.9 \mathrm{VS}$ $29.1 \pm 6.2, P<0.001)$, duration of gout ( $12.6 \pm 4.8$ VS $4.5 \pm 4.8, P<0.001)$, number of involved joints $(12.5 \pm 9.3$ VS $4.3 \pm 3.7, P<0.001)$ and prevalence of CKD $(30.0 \%$ VS $10.1 \%, P=0.034$ ), and lower BMI (23.7 \pm 3.7 VS $\left.26.6 \pm 4.1 \mathrm{~kg} / \mathrm{m}^{2}, \mathrm{P}=0.004\right)$. Logistic regression demonstrated that higher disease duration [OR $1.30,95 \% \mathrm{Cl}$ $(1.15,1.46), \mathrm{P}<0.001]$ and lower BMI [OR $0.80,95 \% \mathrm{Cl}(0.67,0.96), \mathrm{P}=0.015]$ were associated with tophi in the early-onset gout patients.

Table 1. Comparison of variables between early-onset group and control group

\begin{tabular}{lccc}
\hline & Early-onset group (N=99) & Control group (N=402) & $\mathrm{P}$ \\
\hline Age, years & $30.4 \pm 8.2$ & $57.9 \pm 13.5$ & $<0.001$ \\
Female, \% & $1.0 \%$ & $12.7 \%$ & $<0.001$ \\
Age of onset, years & $24.2 \pm 5.8$ & $51.7 \pm 13.9$ & $<0.001$ \\
Duration of gout, years & $6.2 \pm 5.8$ & $6.2 \pm 5.6$ & 0.953 \\
Frequency of flare in last year & $6.4 \pm 7.5$ & $9.3 \pm 10.9$ & 0.022 \\
sUA, mg/dl & $10.6 \pm 2.4$ & $8.9 \pm 2.5$ & $<0.001$ \\
>10mg/dl, \% & $59.6 \%$ & $32.8 \%$ & $<0.001$ \\
No. of involved joints & $5.9 \pm 6.0$ & $5.9 \pm 5.5$ & 0.981 \\
Tophi, \% & $20.2 \%$ & $26.1 \%$ & 0.397 \\
Alcohol overuse, \% & $35.4 \%$ & $34.1 \%$ & 0.811 \\
BMI, kg/m² & $26.1 \pm 4.2$ & $24.8 \pm 3.9$ & 0.004 \\
Obesity, \% & $27.3 \%$ & $15.5 \%$ & 0.005 \\
Dyslipidemia, \% & $51.5 \%$ & $54.0 \%$ & 0.666 \\
Hypertension, \% & $14.1 \%$ & $44.0 \%$ & $<0.001$ \\
DM, \% & $6.1 \%$ & $24.6 \%$ & $<0.001$ \\
Urolithiasis, \% & $19.2 \%$ & $33.6 \%$ & 0.007 \\
eGFR, ml/min $/ 1.73 m^{2}$ & $84.8 \pm 51.0$ & $62.9 \pm 19.7$ & $<0.001$ \\
CKD, \% & $14.1 \%$ & $45.6 \%$ & $<0.001$ \\
\hline
\end{tabular}

sUA: serum uric acid; BMI: body mass index; DM: diabetic mellitus; eGFR: estimated glomerular filtration rate; CKD: chronic kidney disease.

Conclusions: Early-onset gout exhibited higher sUA and was associated with obesity but not with hypertension, diabetic mellitus and renal insufficiency. Longer disease duration and lower BMI were associated with tophi in the early-onset gout patients.

Acknowledgements: The present study was supported by Guangdong Natural Science Foundation, China (Grant no. 2014A030310086) to Qian-Hua Li.

Disclosure of Interest: None declared

DOI: 10.1136/annrheumdis-2017-eular.6307

\section{THU0440 TEMPORAL TRENDS OF VENOUS THROMBOEMBOLISM RISK BEFORE AND AFTER DIAGNOSIS OF GOUT. A GENERAL POPULATION-BASED STUDY}

L. Li ${ }^{1,2}$, N. McCormick ${ }^{1,2}$, E.C. Sayre ${ }^{1}$, J. Esdaile ${ }^{1,2}$, D. Lacaille ${ }^{1,2}, H . X^{1,3}$ H.K. Choi ${ }^{1,4}$, J.A. Avina-Zubieta ${ }^{1,2} .{ }^{1}$ Arthritis Research Canada, Richmond; ${ }^{2}$ The University of British Columbia, Vancouver; ${ }^{3}$ Simon Fraser University, Burnaby, Canada; ${ }^{4}$ Harvard Medical School, Boston, United States

Background: Data on the risk of venous thromboembolism (VTE, including deep venous thrombosis and pulmonary embolism) in patients with gout are scarce.

Objectives: 1) To estimate the overall risk of VTE in an incident gout cohort; 2) To estimate the temporal trends of VTE before and after gout diagnosis compared with the general population.

Methods: We conducted a matched cohort study using a population based administrative health database that includes all outpatient visits, hospital admissions, vital statistics, and dispensed medications for all residents in British Columbia, Canada. Gout cases were defined using one ICD-9/10 code (274/M10) from a 